rotation $0.89(0.37 ; 1.40))$. Clinical tests indicated increased shoulder stiffness.

Conclusions The shoulder strengthening programme was feasible in patients with HSD and long-lasting shoulder symptoms. A future randomised controlled trial, with an improved recruitment strategy, will demonstrate whether the exercise programme is effective in improving symptoms in this patient group.

\section{SONOGRAPHIC TENDON ABNORMALITIES CAN PREDICT SYMPTOMATIC TENDINOPATHY IN MARATHON RUNNERS}

Sarah Eby, Masaru Teramoto, Rudi Zurbuchen, Keith Cummings, Joy English, Daniel M Cushman. University of Utah, Salt Lake City, USA

\subsection{6/bjsports-2021-I0C.36}

Background Tendinopathies of the patellar and Achilles tendons are common injuries among athletes and the general population. These injuries are costly, time-intensive to treat, and significantly limit activity. Predicting and eventually preventing these injuries is invaluable.

Objective Evaluate the relationship between sonographic tendon abnormalities and development of future injury in community marathon runners.

Design Prospective longitudinal cohort study. A blinded reviewer rated pre-race ultrasound images for tendon abnormalities, including thickening, hypoechogenicity, intratendinous delamination, or calcifications.

Setting 2019 Salt Lake City Marathon

Participants Marathon or half-marathon runners with no knee or ankle symptoms. 166 were screened; 138 completed the study $(36.2 \pm 12.0$ years of age, male: $n=68)$.

Assessment of Risk Factors Ultrasound evaluation of bilateral Achilles and patellar tendons immediately prior to the race and longitudinal surveys of subsequent injury development immediately following the race, and 1 and 3 months postrace.

Main Outcome Measurements Patellar or Achilles injury development. Statistical analyses included calculating relative risk (RR) with a 95\% confidence interval (CI) and hazard ratio (HR) with a 95\% CI from the Cox proportional hazards $(\mathrm{PH})$ model.

Results Sonographic abnormalities were found among $12.3 \%$ (left Achilles) and 26.8\% (right patellar) of runners. Runners with sonographic abnormalities were 2 times $(\mathrm{RR}=2.20$, $95 \% \mathrm{CI}=1.15,4.22, p=.040)$ more likely to develop pain in the Achilles tendon within three months compared to those with normal ultrasounds. The Cox $\mathrm{PH}$ model indicated that sonographic abnormalities were associated with 2 times (HR $=2.35,95 \% \mathrm{CI}=1.10,4.99, p=.027$ ) higher risk of developing post-race pain in the Achilles tendon after adjusting for runners' demographics and training regimens. No significant findings were observed between sonographic abnormality and post-race pain in the patellar tendon for the current observation period.

Conclusions Pre-race sonographic Achilles tendon abnormalities are associated with development of Achilles tendon pain within 3 months. Subsequent injury development, including at 6,9 , and 12-months post-race is pending.

\section{SHOULDER ROTATION STRENGTH CHANGES FROM PRESEASON TO MIDSEASON: A COHORT STUDY OF 292 YOUTH ELITE HANDBALL PLAYERS WITHOUT SHOULDER PROBLEMS}

${ }^{1}$ Behnam Liaghat, ${ }^{2}$ Jesper Bencke, ${ }^{3}$ Mette Kreutzfeldt Zebis, ${ }^{4}$ Henrik Sørensen, ${ }^{5}$ Grethe Myklebust, ${ }^{6}$ Niels Wedderkopp, ${ }^{7}$ Martin Lind, ${ }^{1}$ Merete Møller. ${ }^{1}$ Research Unit for Musculoskeletal Function and Physiotherapy, Department of Sports Science and Clinical Biomechanics, University of Southern Denmark, Odense, Denmark; ${ }^{2}$ Human Movement Analysis Laboratory, Copenhagen University Hospital, Amager-Hvidovre, Copenhagen, Denmark; ${ }^{3}$ Department of Physiotherapy, Faculty of Health and Technology, University College Copenhagen, Copenhagen, Denmark; ${ }^{4}$ Department of Public Health, Section for Sport Science, Aarhus University, Aarhus, Denmark; ${ }^{5}$ Department of Sports Medicine, Oslo Sports Trauma Research Center, Norwegian School of Sport Sciences, Oslo, Norway; ${ }^{6}$ Department of Regional Health Research, University of Southern Denmark, Odense, Denmark; ' Div. of Sportstraumatology, Aarhus University Hospital, Aarhus, Denmark

\subsection{6/bjsports-2021-IOC.37}

Background Shoulder rotation strength deficit measured at one time-point during preseason has been investigated as a risk factor for shoulder problems in sports with conflicting results. However, athletes face changes in physical demands and accumulative training exposure during a season, which likely influence their rotation strength over this period.

Objective We aimed to investigate if shoulder rotation strength changes during a competitive season in a cohort of youth elite handball players.

Design Prospective cohort study.

Setting Danish youth elite handball clubs.

Patients (or Participants) Players ( $\mathrm{n}=292,45 \%$ girls, 14-18 years) without shoulder problems were assessed at preseason and at midseason.

Interventions (or Assessment of Risk Factors) We measured isometric internal rotation (IR) and external rotation (ER) strength in supine with the shoulder abducted $90^{\circ}$ in neutral rotation and $30^{\circ}$ of IR measured using hand-held dynamometry, and the corresponding ER/IR ratios.

Main Outcome Measurements Changes in shoulder strength and ER/IR strength ratios.

Results From preseason to midseason, ER/IR mean ratios were higher in neutral rotation (boys difference 0.02 , 95\% CI 0.00 to 0.05 ; girls difference 0.05 , 95\% CI 0.010 .09 ) and in $30^{\circ}$ IR (boys difference 0.15 , 95\% CI 0.11 to 0.19 ; girls difference 0.12 , 95\% CI $0.07-0.17$ ) due to an increase in ER strength and a decrease in IR strength.

Conclusions The present finding supports that shoulder strength ratios change between two time points during a competitive season. Clinicians and researchers should be aware that strength in youth elite handball players may have a normal variation over the course of the season. This warrants ongoing monitoring and should be considered when normative reference values are compared, and when measuring the effect of targeted exercise programmes. Studies investigating if shoulder strength is a causal factor for shoulder problems should consider including the strength variable as a time-varying covariate. 